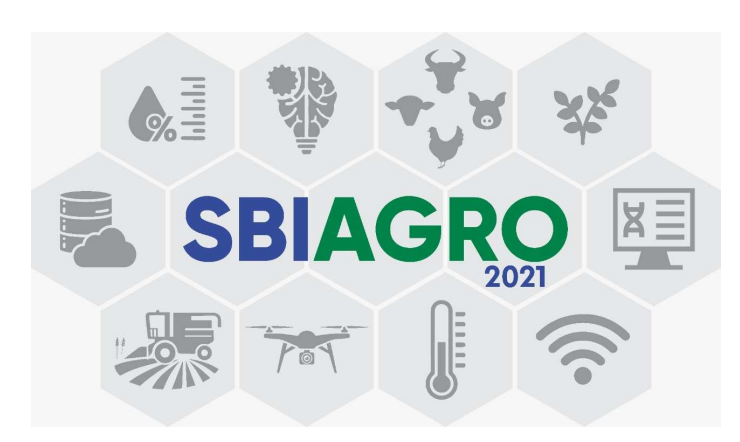

\title{
Estimating and tuning adaptive action plans for the control of smart interconnected poultry houses
}

\author{
Darlan F Klotz $^{1}$, Dalcimar Casanova ${ }^{1}$, Marcelo Teixeira ${ }^{1}$ \\ ${ }^{1}$ Graduate Program in Electrical Engineering \\ Federal University of Technology Paraná \\ Pato Branco - PR - Brazil \\ darlan.klotz@ifc.edu.br, \{dalcimar,mtex\}@utfpr.edu.br
}

\begin{abstract}
In poultry farming, the systematic choice, update, and implementation of periodic $(t)$ action plans define the feed conversion rate $\left(F C R^{\langle t\rangle}\right)$, which is an acceptable measure for successful production. Appropriate action plans provide tailored resources for broilers, allowing them to grow within the socalled comfort zone, without waste or lack of resources. Although the implementation of an action plan is automatic, its configuration depends on a specialist, which tends to be inefficient and result in variable $F C R^{\langle t\rangle}$. In this project, the specialist's perception is reproduced, to some extent, by computational intelligence. By combining deep learning and genetic algorithm techniques, we show how action plans can adapt their performance over the time, based on previous well succeeded plans. We also implement a network infrastructure to replicate our method over distributed poultry houses, for their smart, interconnected, and adaptive control. A supervision system is provided as interface to users. Experiments using real data suggest an improvement of $5 \%$ on the performance of the most productive specialist, staying close to the optimal $F C R^{\langle t\rangle}$.
\end{abstract}

\section{Introduction}

Poultry farming is the agro-industrial sector focused on producing broiler meat for consumption. Worldwide, the activity has attracted attention due to its economic and social role, aligned with the continuously increasing world demand for broilers. Only in 2019 the USA was responsible for producing 19.941 million tons of meat, followed by Brazil and China with 13.245 and 13.750 million tons, respectively (ABPA 2021).

Technically, broilers raise within poultry houses ( $\mathrm{PHs}$ ), that are automatically controlled by electronic devices. The daily recipe, prescribing the parameters for the automatic controller, is called an action plan (Acres 2020). The quality of an action plan reflects the general welfare for broilers, allowing them to grow up under appropriate conditions of feeding, housing, and health. In this paper, in particular, an action plan is 
exploited in terms of its environment-related variables. We seek for combined indoor climate conditions that allow broilers to grow up under thermal comfort, achieved within the so-called comfort zone (Botreau et al. 2007). The comfort zone directly affects productivity, which is acceptably measured by periodic $(t \in \mathbb{N})$ feed conversion rates $\left(F C R^{\langle t\rangle}\right)$ estimations. The more accurate the action plan, the lower the accumulated $F C R^{\langle t\rangle}$, and the greater it tends to be the production.

Advances on improving $F C R^{\langle t\rangle}$ focus on health, nutrition, genetics, thermal comfort (Henriksen et al. 2016, Ribeiro et al. 2019), etc. A remaining barrier is, however, the lack of expert systems to help humans with the choice, update, and implementation of daily action plans. This leads specialists to use their own experience to set up action plans, which tends to be complex, inefficient, and prone to errors, besides to cause conflicting opinions. As a consequence of unsystematic interventions, each $\mathrm{PH}$ may return a distinct $F C R^{\langle t\rangle}$, which makes unclear the overall performance estimation.

The literature provides alternatives to estimate intelligent action plans. For example, (Ribeiro et al. 2019) uses a bio-inspired model based on Artificial Neural Networks (ANNs) to suggest action plans according to climatic variations. However, this approach is not integrated with the process controller, so that it cannot reconfigure its actions dynamically according to the ANN suggestion. Furthermore, (Ribeiro et al. 2019) does not consider the dynamic adaptation of its estimations over the time, which is an important feature when scenarios of production are variable.

In this project, the specialist perception is reproduced, to some extent, by computational intelligence. Differently from (Ribeiro et al. 2019), we use a more suitable memory-aware model based on Long short-term memory (LSTM) networks, instead of ANNs. We also show how estimated action plans can be combined across algorithms that artificially emulate genetic mutations. This results in improved action plans that are capable of adapting themselves over the time based on previously successful action plans. By replicating our method over a set of PHs, we solidify a new foundation to support smart, cooperative, distributed, and interconnected poultry farming.

Experiments conducted using real data indicate that our approach estimates an $F C R^{\langle 40\rangle}$ of 1.5610 by the end of a flock. In comparison, the $F C R^{\langle 40\rangle}$ resulting from the progressive application of empiric action plans, chosen by the specialist who had the best performance among all others in the evaluated group, under the same setup, was of 1.640, therefore 0.079 or $5 \%$ worse than ours. Our method also shows proximity with the best performance achievable by Ross broilers strain, which is $F C R^{\langle 40\rangle}=1.558$ (Ross 2019).

\section{An intelligent solution for poultry management}

A usual measure to quantify the efficiency in transforming feed into animal protein, is based on periodic samples of Feed Conversion Rate, formalised as:

$$
F C R^{\langle t\rangle}=\frac{F C^{\langle t\rangle}}{L B^{\langle t\rangle} \cdot M W^{\langle t\rangle}}
$$

where $t$ is an integer associated with the day of observation; $F C^{\langle t\rangle}$ is the amount of feed consumption until day $t$; $L B^{\langle t\rangle}$ is the number of living birds at day $t$; and $M W^{\langle t\rangle}$ is the mean weigh at day $t$. 
An effective action plan is achieved when the $F C R^{\langle t\rangle}$ is improved, i.e., when it approximates to zero. The more accurate the action plan, the lower the conversion rate, and the greater the production tends to be. However, obtaining a satisfactory $F C R^{\langle t\rangle}$, at the end of a flock (usually $t=40$ ), requires enormous ability from the specialist to conduct the daily management. It depends, basically, on how the daily parameters are informed to the controller so that, in combination, they could result in a reasonable accumulated $F C R^{\langle 40\rangle}$ for the entire flock.

In general, poultry farmers handle more than one $\mathrm{PH}$ within a shared infrastructure, called a poultry condominium (PC), shown in Fig. 1 and formalised as a finite set Cond $=\left\{P H_{1}, \cdots, P H_{n}\right\}$ of poultry houses $P H_{i}$, for $1 \leq i \leq n$.
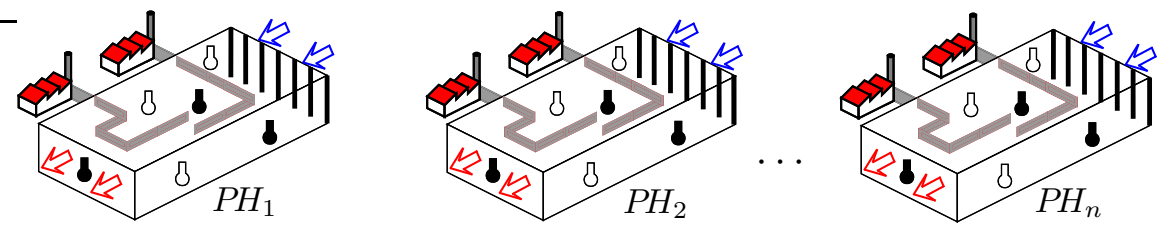

Figure 1. Structure poultry house condominium Cond $=\left\{P H_{1}, \cdots, P H_{n}\right\}$.

Each PH has automated feed and drinking systems working continuously from 6 AM until 10 PM. It also includes electronic devices to inform the daily feed consumption $\left(F C^{\langle t\rangle}\right)$, and the mean weight $\left(M W^{\langle t\rangle}\right)$ captured by automatic scales. Each flock takes about 40 days to be produced ( $t=1, \cdots, 40$ ), and the final weight (i.e, $M W^{\langle 40\rangle}$ ) is expected to be of approximately $2.8 \mathrm{Kg}$ per bird. Temperature $\left(\operatorname{Min}(T)^{\langle t\rangle}, \operatorname{Avg}(T)^{\langle t\rangle}\right.$, and $\left.\operatorname{Max}(T)^{\langle t\rangle}\right)$, and relative humidity $\left(\operatorname{Min}(H)^{\langle t\rangle}, \operatorname{Avg}(H)^{\langle t\rangle}\right.$, and $\operatorname{Max}(H)^{\langle t\rangle}$ ), inside the $\mathrm{PH}$ are collect by sensors and stored into a database, which is part of the control system infrastructure. The specialist also records the mortality on the day $t\left(M^{\langle t\rangle}\right)$, which alternatively can be exposed as the number of living broilers by:

$$
L B^{\langle t\rangle}=|F|-\sum_{t=1}^{p} M^{\langle t\rangle},
$$

i.e., as the difference between the initial amount of birds in a flock $(|F|)$, and the accumulated mortality recorded by the specialist during a period $p$.

\subsection{Dataset acquisition}

For the purpose of this paper, the real dataset was obtained by observing a PC composed of three PHs, which were used for collecting daily data sampling. The three PHs contained only male, Ross strain broilers. The data acquisition was conducted between August 2019 and March 2020, alternating therefore different seasonal features. A total of 12 flocks were observed (i.e., 12 samples), the same as used in related literature (Johansen et al. 2017), which has shown to be sufficient for the intended analysis.

Table 1 shows the variables collected and used in the experiments that follow. Only input and output variables have been formalised so far, while normalised variables will be introduced in section Section 2.2. Input variables correspond to the action plan for the day $t$, while output variables are those influenced by this action plan. The selection algorithm Greedy Stepwise (Gevrey et al. 2003) has been applied to confirm the relevance of the input variables over the output. 


\begin{tabular}{r|r|r}
\hline Input variables & Unit & Notation \\
\hline day & $t=1, \cdots, 40$ & $t$ \\
\hline minimum temperature & {$\left[{ }^{\circ} \mathrm{C}\right]$} & $\operatorname{Min}(T)^{\langle t\rangle}$ \\
mean temperature & {$\left[{ }^{\circ} \mathrm{C}\right]$} & $\operatorname{Avg}(T)^{\langle t\rangle}$ \\
maximum temperature & {$\left[{ }^{\circ} \mathrm{C}\right]$} & $\operatorname{Max}(T)^{\langle t\rangle}$ \\
\hline minimum humidity & {$[\%]$} & $\operatorname{Min}(H)^{\langle t\rangle}$ \\
mean humidity & {$[\%]$} & $\operatorname{Avg}(H)^{\langle t\rangle}$ \\
maximum humidity & {$[\%]$} & $M a x(H)^{\langle t\rangle}$ \\
\hline Output variables & & Notation \\
\hline mean daily weigh & {$[g]$} & $M W^{\langle t\rangle}$ \\
daily feed consumption & {$[\mathrm{Kg}]$} & $F C^{\langle t\rangle}$ \\
number of living birds & {$[\mathrm{birds}]$} & $L B^{\langle t\rangle}$ \\
daily mortality & {$[\mathrm{birds}]$} & $M^{\langle t\rangle}$ \\
\hline Output variables normalized & & $\mathrm{Notation}^{\langle t\rangle}$ \\
\hline daily feed consumption per bird & {$[\mathrm{Kg} / \mathrm{bird}]$} & $F C \mathrm{~b}^{\langle t\rangle}$ \\
number of living birds per area & {$\left[\right.$ bird $\left./ \mathrm{m}^{2}\right]$} & $L B \mathrm{a}^{\langle t\rangle}$ \\
daily mortality per area & {$\left[\right.$ bird $\left./ \mathrm{m}^{2}\right]$} & $\mathrm{Ma}^{\langle t\rangle}$ \\
\hline
\end{tabular}

Table 1. Variables to be considered in our experiments.

A daily measurement was collected for each of the 7 input variables in Table 1 , totalling 280 records $(7 \times 40$ days of the flock) in one sample. For the 3 output variables, the daily measurement for $M W^{\langle t\rangle}, F C^{\langle t\rangle}$, and $L B^{\langle t\rangle}$ corresponds to 120 records $(3 \times 40)$, also for one sample. Therefore, by observing $3 \mathrm{PHs}$, each one providing 4 samples, we obtained the total of 12 samples, corresponding to an input data matrix of $12 \times 280$, while the corresponding output data form a matrix sized $12 \times 120$.

\subsection{Data normalisation}

The 3 observed PHs occupy two different areas. $A_{P H_{1}}$ is $150 \mathrm{~m}$ long by $16 \mathrm{~m}$ wide, totalling $2400 \mathrm{~m}^{2}$, and it can host about 34800 birds. $A_{P H_{2}}$ is $150 \mathrm{~m}$ long by $12 \mathrm{~m}$ wide, with a floor area of $1800 \mathrm{~m}^{2}$ that accommodates 26500 birds. Thus, $M^{\langle t\rangle}$ and $L B^{\langle t\rangle}$ can be normalised by the area $A_{P H_{i}}$, such that:

$$
M \mathrm{a}^{\langle t\rangle}=\frac{M^{\langle t\rangle}}{A_{P H_{i}}} ; \quad \text { and } \quad L B \mathrm{a}^{\langle t\rangle}=\frac{L B^{\langle t\rangle}}{A_{P H_{i}}} .
$$

Also $F C^{\langle t\rangle}$ can be normalised by $L B^{\langle t\rangle}$, given the feed consumption per bird:

$$
F C \mathrm{~b}^{\langle t\rangle}=\frac{F C^{\langle t\rangle}}{L B^{\langle t\rangle}}
$$

In this way, $F C R^{\langle t\rangle}$ can result from Eq11 additionally pondering: (i) the number of living birds per area $A_{P H_{i}}\left(L B \mathrm{a}^{\langle t\rangle}\right)$; and (ii) the daily feed consumption by the number of living birds $\left(F C \mathrm{~b}^{\langle t\rangle}\right)$. Eq.5 formalises this idea:

$$
F C R^{\langle t\rangle}=1000 \frac{F C \mathrm{~b}^{\langle t\rangle}}{L B \mathrm{a}^{\langle t\rangle} M W^{\langle t\rangle}} L B \mathrm{a}^{\langle t\rangle}
$$

where 1000 compensates the weight conversion from $K g$ to $g$. As Mean $(\mu)$ and standard deviation $(\sigma)$ are essential measures, they are detailed for the 12 flocks in Fig. 2 . 


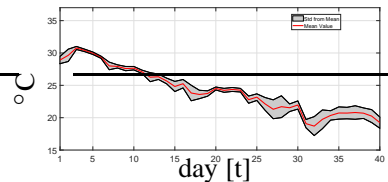

(a) Input: $\operatorname{Min}(T)^{\langle t\rangle}$

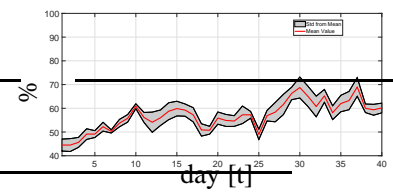

(d) Input: $\operatorname{Min}(H)^{\langle t\rangle}$

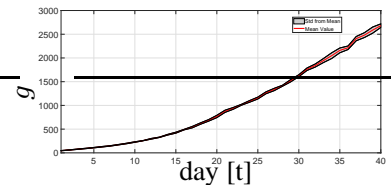

(g) Output: $M W^{\langle t\rangle}$

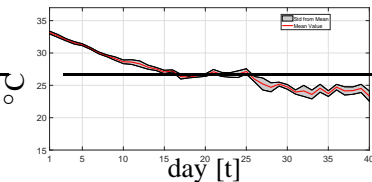

(b) Input: $\operatorname{Avg}(T)^{\langle t\rangle}$

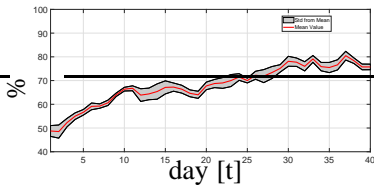

(e) Input: $\operatorname{Avg}(H)^{\langle t\rangle}$

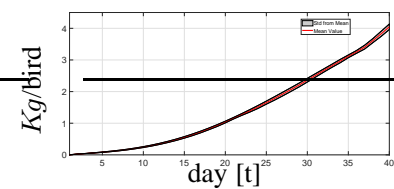

(h) Output: $F C b^{\langle t\rangle}$

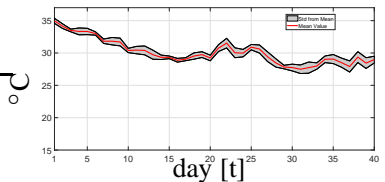

(c) Input: $\operatorname{Max}(T)^{\langle t\rangle}$

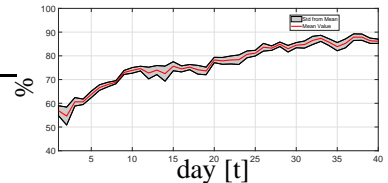

(f) Input: $\operatorname{Max}(H)^{\langle t\rangle}$

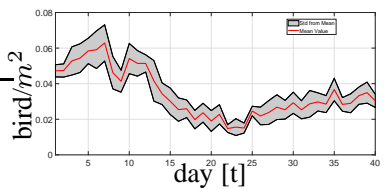

(i) Output: $M a^{\langle t\rangle}$

Figure 2. Statistical view of the collected variables.

\subsection{Proposed LSTM Neural Network}

Now, we feed the LSTM neural network shown in Fig. 3 with: the day under analysis $(t)$; the measured temperature $\left(\operatorname{Min}(T)^{\langle t\rangle}, \operatorname{Avg}(T)^{\langle t\rangle}\right.$, and $\left.\operatorname{Max}(T)^{\langle t\rangle}\right)$ and humidity $\left(\operatorname{Min}(H)^{\langle t\rangle}, \operatorname{Avg}(H)^{\langle t\rangle}\right.$, and $\left.\operatorname{Max}(H)^{\langle t\rangle}\right)$; and the previous day mean weigh $\left(M W^{\langle t-1\rangle}\right)$, feed consumption per bird $\left(F C \mathrm{~b}^{\langle t-1\rangle}\right)$, and number of living birds per area $\left(L B \mathrm{a}^{\langle t-1\rangle}\right)$.

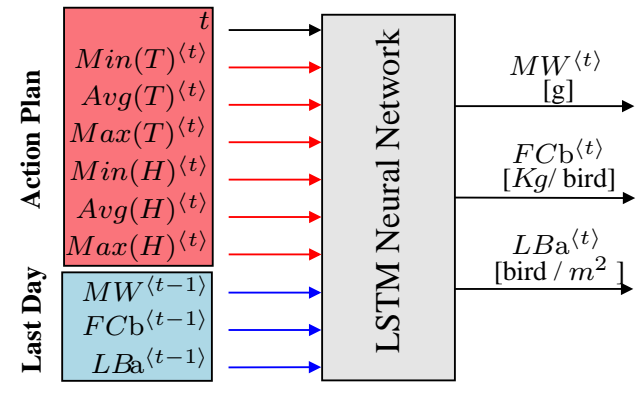

Figure 3. Proposed LSTM network architecture.

From now forward, inputs and outputs are referred to as vectors: $\mathbf{x}^{\langle t\rangle}=$ $\left[t, \operatorname{Min}(T)^{\langle t\rangle}, \cdots, \operatorname{Max}(H)^{\langle t\rangle}\right] ; \mathbf{h}^{\langle t-1\rangle}=\left[M W^{\langle t-1\rangle}, F C \mathrm{~b}^{\langle t-1\rangle}, L B \mathrm{a}^{\langle t-1\rangle}\right] ; \mathbf{h}^{\langle t\rangle}=$ $\left[M W^{\langle t\rangle}, F C \mathrm{~b}^{\langle t\rangle}, L B \mathrm{a}^{\langle t\rangle}\right]$. Vector $\mathbf{x}^{\langle t\rangle}$ carries the input action plan for day $t ; \mathbf{h}^{\langle t-1\rangle}$ is the input vector with the previous days outputs; and $\mathbf{h}^{\langle t\rangle}$ is the output vector for day $t$. By concatenating $\mathbf{x}^{\langle t\rangle}$ and $\mathbf{h}^{\langle t-1\rangle}$ (denoted $\mathbf{x}^{\langle t\rangle} \frown \mathbf{h}^{\langle t-1\rangle}$ ), and by applying it as the neural network input, we obtain a prediction for $\mathbf{h}^{\langle t\rangle}$. The data pre-processing step and associated hyperparameters settings for training the LSTM model can be found in (Klotz et al. 2020).

\subsection{LSTM Implementation}

The proposed LSTM-based architecture can be implemented as in Algorithm 1, and then it is taken as a fitness function of a GA in Algorithm 2 .

After 1400 iterations the proposed algorithm starts to stabilise. This leads search to stop by the timeout criterion with no changes in the fitness function. The $F C R^{\langle 40\rangle}$ 

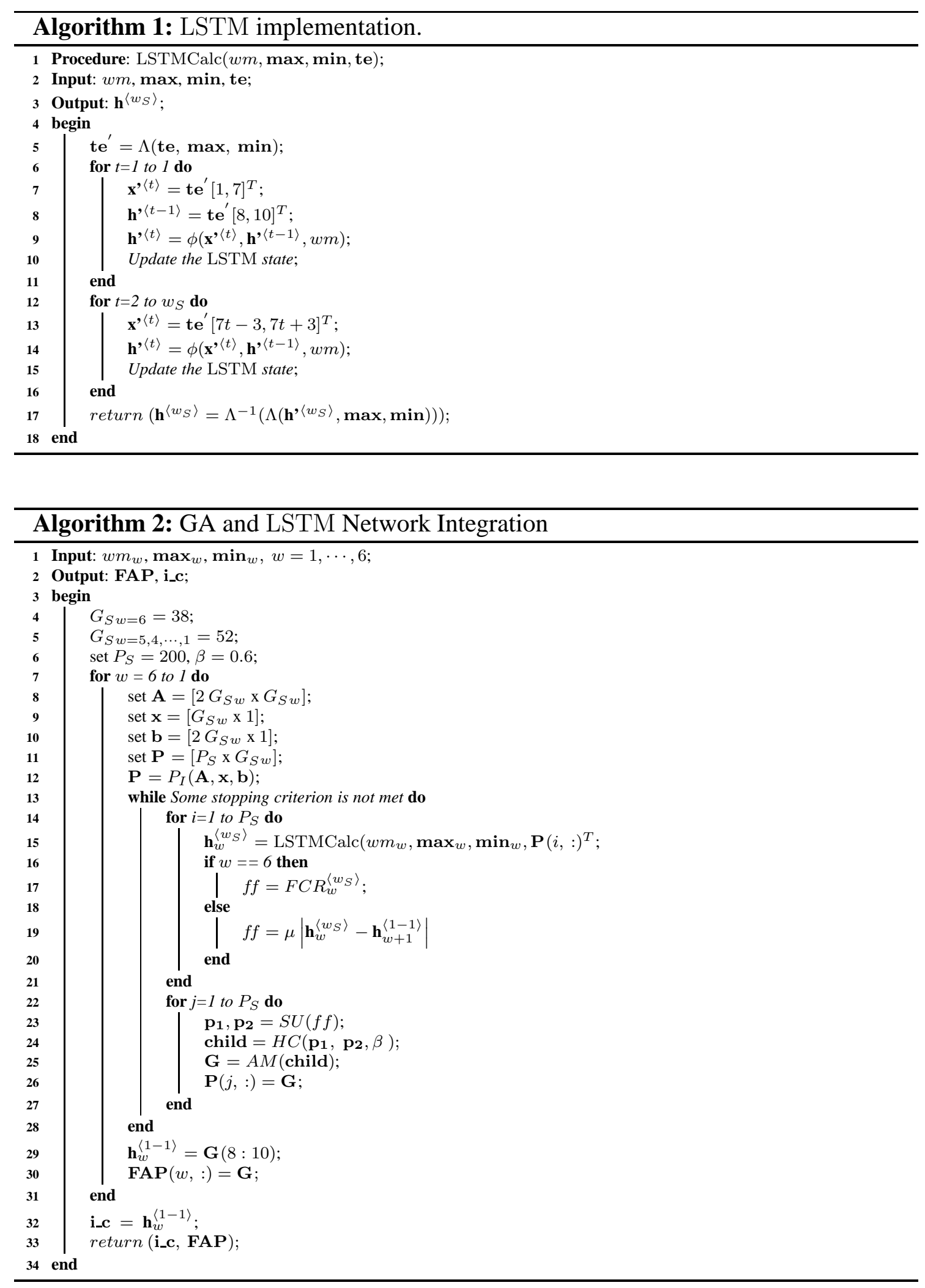

upon stabilisation is 1.5602 . However, this value is exclusively linked to $w m_{6}$, i.e., as the Algorithm 2 searches from $w m_{6}$ to $w m_{1}$, the value of 1.5602 is guaranteed if and only if the progressive error between the difference of $\mathbf{h}_{w}^{\left\langle w_{S}\right\rangle}$ (estimated by the $w m_{w}$ model) and 
$\mathbf{h}_{w+1}^{\langle 1-1\rangle}$ (of the model $w m_{w+1}$ ) is null (see Algorithm 2, line 19). The following Table 2 shows step-by-step these errors.

\begin{tabular}{c|ccc|ccc}
\hline \multirow{3}{*}{$w$} & \multicolumn{5}{|c}{$\left|\mathbf{h}_{w}^{\left\langle w_{S}\right\rangle}-\mathbf{h}_{w+1}^{\langle 1-1\rangle}\right|$} \\
\cline { 2 - 7 } & \multicolumn{3}{|c|}{ Error } & \multicolumn{3}{|c}{ Relative Error (\%) } \\
\cline { 2 - 7 } & $M W^{\langle t\rangle}$ & $F C \mathrm{~b}^{\langle t\rangle}$ & $L B \mathrm{a}^{\langle t\rangle}$ & $M W^{\langle t\rangle}$ & $F C \mathrm{~b}^{\langle t\rangle}$ & $L B \mathrm{a}^{\langle t\rangle}$ \\
\hline 5 & 17.4840 & 0.0066 & 0.1448 & 0.8335 & 0.2230 & 1.0269 \\
4 & 32.7932 & 0.0011 & 0.0092 & 2.3440 & 0.1960 & 0.0639 \\
3 & 15.7567 & 0.0070 & 0.1289 & 1.7667 & 0.7056 & 0.9059 \\
2 & 0.0000 & 0.0080 & 0.0765 & 0 & 2.2396 & 0.5358 \\
1 & 1.6142 & 0.0015 & 0.1461 & 1.0338 & 1.4681 & 1.0176 \\
\hline
\end{tabular}

Table 2. Errors and relative erros comparison.

We present in Fig. 4 the action plan FAP returned by the Algorithm 2 after it iterates recurrently through the 6 weeks, leading to $F C R^{\langle E\rangle}=1.5602$. The abscissa axis shows the input $t$ of the LSTM network (see Fig.3). The ordinate axis shows the inputs $\operatorname{Min}(T)^{\langle t\rangle}, \operatorname{Avg}(T)^{\langle t\rangle}$, and $\operatorname{Max}(T)^{\langle t\rangle}$ (Fig. 4(a)); and the inputs $\operatorname{Min}(H)^{\langle t\rangle}, \operatorname{Avg}(H)^{\langle t\rangle}$, and $\operatorname{Max}(H)^{\langle t\rangle}$ (Fig. 4(b)) that were discovered by the GA to reach $F C R^{\langle E\rangle}=1.5602$.

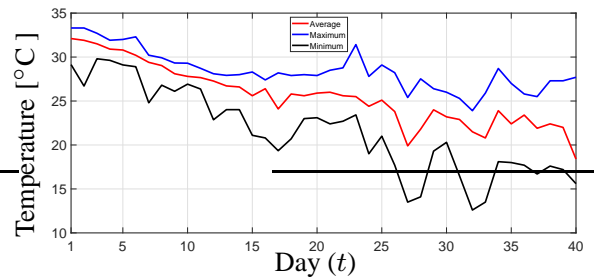

(a) Temperature

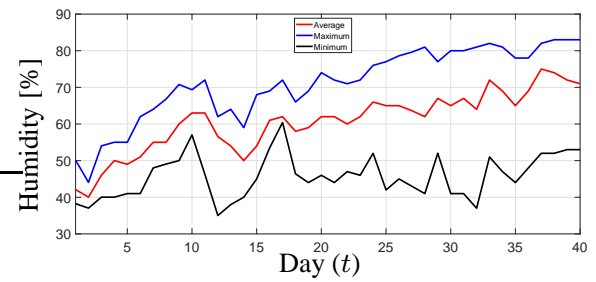

(b) Humidity

Figure 4. Action plan suggested by our approach.

Now, we apply the Algorithm 1 progressively from $w m_{w}=1$ to $w m_{w}=6$, having FAP and i_c as input for each respective model. Upon termination of Algorithm 1. we capture the 3 outputs from the last day of $w m_{6}$ (i.e., $\mathbf{h}_{w=6}^{\left\langle w_{S}\right\rangle}$ ) and apply Eq 5 to obtain $F C R^{\langle R\rangle}$. It is expected a very close relationship between $F C R^{\langle R\rangle}$ and $F C R^{\langle E\rangle}$, considering the statistical correctness of Table 2. For the particular dataset, $F C R^{\langle R\rangle}=$ 1.5610 was found for the 40-day flock, therefore very close to $F C R^{\langle E\rangle}=1.5602$.

\subsubsection{Quantitative analysis}

In order to assess how competitive our estimations are, we compare the model performance $\left(F C R^{\langle R\rangle}\right.$ ) against: (i) the optimal performance (denote $F C R^{\langle O\rangle}$ ) theoretically expected for the Ross broiler strain; (ii) the performance resulting from real managements applied by human specialists; (iii) the performance resulting from 1000 other candidate, artificially created, action plans.

The optimal performance for Ross broiler strain after 40 days is $F C R^{\langle O\rangle}=1.558$ (Ross 2019). Therefore, the $F C R^{\langle R\rangle}=1.5610$, resulting from our method, suggests an approximation only $0.192 \%$ worse than $F C R^{\langle O\rangle}$. The best specialist (denote $F C R^{\langle X\rangle}$ ), among all others, returned $F C R^{\langle X\rangle}=1.640$, therefore 0.0790 (or $5 \%$ ) worse than $F C R^{\langle R\rangle}=1.5610$. Although the difference seems to be minor, our model allows to 
save $0.0790 \mathrm{Kg}$ (or $79 \mathrm{~g}$ ) of feed per bird, for each $1 \mathrm{Kg}$ of produced meat. For a slaughtering weight of $2.8 \mathrm{Kg}$, each broiler requires $221.20 \mathrm{~g}$ less feed to be produced. For a PH with about 34000 broilers, this means $7520,8 \mathrm{Kg}$ of feed saving during a single flock.

We further construct 1000 more synthetic action plans to simulate different specialists handling a flock. The best possible performance found was $F C R^{\langle S\rangle}=1.5614$, therefore still worse than $F C R^{\langle R\rangle}=1.5610$.

\section{Conclusion}

In this paper, deep learning and genetic algorithms are combined to estimate action plans for poultry farming. We exploit a production model that interconnects multiple poultry houses and allows us to both, collect distributed data, and propagate optimised action plans throughout a network infrastructure, commanded by a central supervision system.

We show that the specialist perception about the process can be captured and reproduced, to some extent, by using a bio-inspired method, and this may increase productivity. A software solution is also provided and fits with any number of poultry houses, including one, when this is a particular case.

Ongoing researches focus on technology transfer and tests in real scale. Other results are expected from the observation of a more complete set of events related to poultry business, such as cost, profit, and market demand. Environment forecasts and energy consumption are also subjects to be approached in future extensions.

\section{References}

ABPA (2021). Brazilian association of animal protein. EMBRAPA.

Acres, A. (2020). Broiler management handbook.

Botreau, R., Bracke, M., Perny, P., Butterworth, A., Capdeville, J., Van Reenen, C., and Veissier, I. (2007). Aggregation of measures to produce an overall assessment of animal welfare. Animal, 1(8):1188-1197.

Gevrey, M., Dimopoulos, I., and Lek, S. (2003). Review and comparison of methods to study the contribution of variables in artificial neural network models. Ecological modelling, 160(3):249-264.

Henriksen, S., Bilde, T., and Riber, A. B. (2016). Effects of post-hatch brooding temperature on broiler behavior, welfare, and growth. Poultry Science, 95(10):2235-2243.

Johansen, S. V., Bendtsen, J. D., Martin, R., Mogensen, J., et al. (2017). Data driven broiler weight forecasting using dynamic neural network models. IFAC-PapersOnLine, 50(1):5398-5403.

Klotz, D. F., Ribeiro, R., Enembreck, F., Denardin, G., Barbosa, M., Casanova, D., and Teixeira, M. (2020). Estimating action plans for smart poultry houses.

Ribeiro, R., Casanova, D., Teixeira, M., Wirth, A., Gomes, H. M., Borges, A. P., and Enembreck, F. (2019). Generating action plans for poultry management using artificial neural networks. Computers and Electronics in Agriculture, 161:131-140.

Ross (2019). 308 and 308 FF performance objectives. 